УДК 004.412

\title{
A Formula for the Mean Length of the Longest Common Subsequence
}

\author{
Sergej V.Znamenskij* \\ Ailamazyan Program Systems Institute of RAS \\ Peter the First, 4, Veskovo village, \\ Pereslavl area, Yaroslavl region, 152021
}

Russia

Received 10.10.2016, received in revised form 10.11.2016, accepted 20.12.2016

The expected value $E$ of the longest common subsequence of letters in two random words is considered as a function of the $\alpha=|A|$ of alphabet and of words lengths $m$ and $n$. It is assumed that each letter independently appears at any position with equal probability. A simple expression for $E(\alpha, m, n)$ and its empirical proof are presented for fixed $\alpha$ and $m+n$. High accuracy of the formula in a wide range of values is confirmed by numerical simulations.

Keywords: longest common subsequence, expected value, LCS length, simulation, asymptotic formula. DOI: 10.17516/1997-1397-2017-10-1-71-74.

\section{Introduction}

The random words of lengths $m$ and $n$ in the alphabet $\alpha$ are also reffered as random symbol sequences. We consider the letter appearance in different positions of words as equally probable and independent events. So for those random sequences the expected value of the longest common subsequence length is a function of $E(m, n, \alpha)$, which reflects the similarity of the original words.

Since the behavior of this function is related to a variety of generic algorithms for fuzzy search and differences identification, it attracts the attention of researchers for a three decades [1]. However, both the use of mathematical apparatus as in $[2,3]$ and numerical modeling (usually with special algorithms) $[4,5,6]$ succeeded to clarify situation only in special cases $m=n$ or $\alpha=2$ (see [7]).

Even for $\alpha=2$ the asymptotic on $\frac{m}{n}$ became clear only resently [8] (just now without detailed proof). Computer calculations $E$ for small $m, n$, in [9] have identified a similar relation for the $\alpha=4$.

The work is intended to the detection and empirical proof of this relation with except of huge $\alpha$ and small $m+n$ cases.

*svz@latex.pereslavl.ru

(C) Siberian Federal University. All rights reserved 


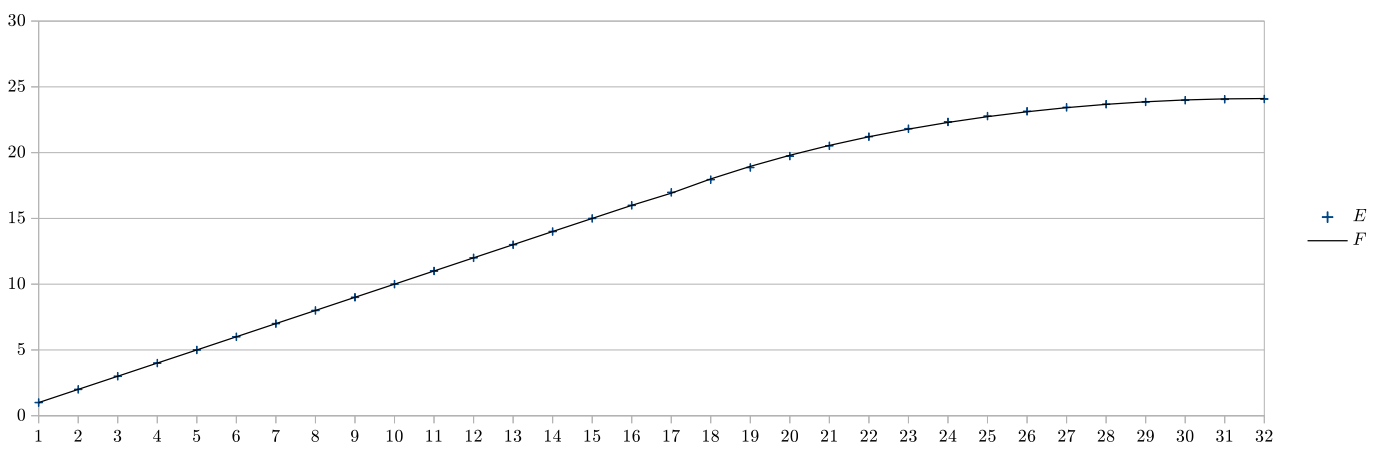

Fig. 1. The case $\alpha=2, n=64$ with of the least relative accuracy

\section{Model}

Hypothesis 1. The functions $r_{x}=r_{x}(\mu, \alpha)$ and $r_{y}=r_{y}(\mu, \alpha)$ exists, for which under the notions $\delta=\frac{m-n}{2 r_{x}}, r=\sqrt{r_{x}^{2}+r_{y}^{2}}$ the function

$$
F(m, n, \alpha)= \begin{cases}n, & 1<r \delta \\ \frac{m+n}{2}-r+r_{y} \sqrt{1-\delta^{2}}, & -1<r \delta<1 \\ m, & r \delta<-1\end{cases}
$$

gives a fine approximation for $E(m, n, \alpha)$ at least for all $\alpha<128$ and $50<m+n<100000$.

\section{Evaluation}

Direct evaluation for huge LCS lengths is impossible due to known square complexity of algorithm. Therefore 6 fixed values of $m+n$ and 10 for $\alpha$ were selected and for $6 \times 10$ series of 32 triplets $(m, n, \alpha)$ their expected values of LSS lengths were calculated as sample means. A perl XS module with a speed compatible with C compiled code was used. Required number of calculations and processed time were detected in a series of runs attempted to get large enough samples for acceptable accuracy. The full collected sample data is available over email to author.

The $r_{x}$ and $r_{y}$ values were calculated which minimizes the mean square error. All the results are presented in the Table 1 . We note $\mathcal{I}_{m, n, \alpha}$ the samle set of all calculated lengths of LCS for generated random words, $\bar{E}(m, n, \alpha)$ their means over each $\mathcal{I}_{m, n, \alpha}$ and calculate their experimental standard deviations

$$
\left.\sigma_{\bar{E}}(s)\right)=\max _{n+m=s, \alpha} \sqrt{\frac{1}{\left|\mathcal{I}_{m, n, \alpha}\right|-1} \sum_{i \in \mathcal{I}_{m, n, \alpha}} l_{i}}, \quad \sigma_{F}(s)=\sqrt{\frac{1}{31} \sum_{j=1}^{32}\left(\bar{E}\left(x_{j, s}, \alpha\right)-F\left(x_{j, s}, \alpha\right)\right)^{2}},
$$

where $x_{j, s}=\left(\frac{j s}{64}, s-\frac{j s}{64}\right)$.

The worst matches from all $6 \times 10$ tests are shown on the Fig. 1 . 
Table 1. The results of empirical proof

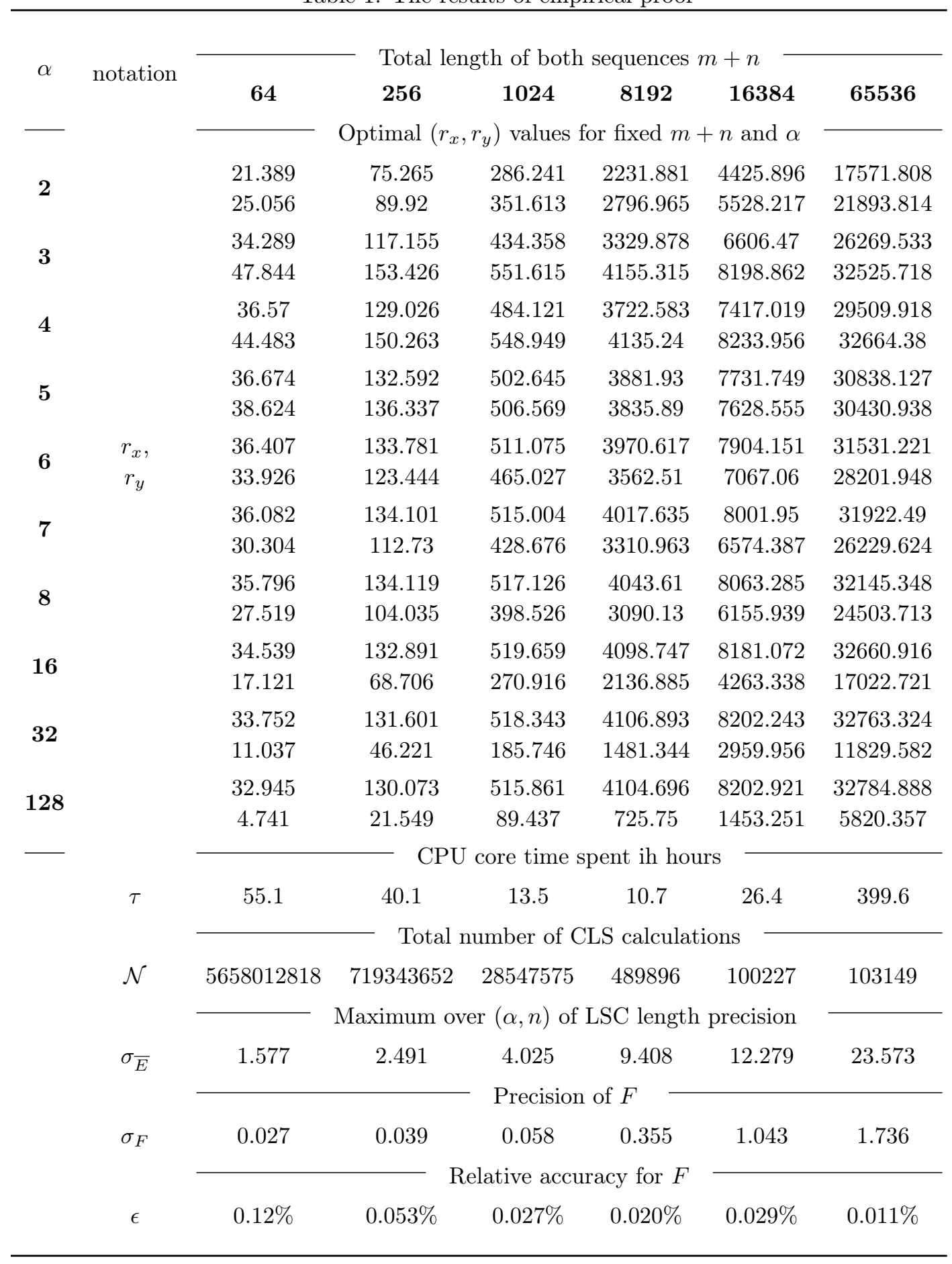




\title{
Acknowledgments
}

This work was performed under financial support from the Government, represented by the Ministry of Education and Science of the Russian Federation (Project ID RFMEFI60414X0138).

\section{References}

[1] V.Chvátal, D.Sankoff, Longest common subsequences of two random sequences, J. Appl. Prob., 12(1975), 306-315.

[2] M.A.Kiwi, M.Loebl, J.Matousek, Expected length of the longest common subsequence for large alphabets, Advances in Mathematics, 197(2005), no. 2, 480-498.

[3] G.S.Lueker, Improved bounds on the average length of longest common subsequences, Journal of the ACM (JACM), 56 (2009), no. 3, 17.

[4] R.Bundschuh, High precision simulations of the longest common subsequence problem, The European Physical Journal B - Condensed Matter and Complex Systems, 22(2001), no. 4, $533-541$.

[5] J.Boutet de Monvel, Extensive simulations for longest common subsequences The European Physical Journal B - Condensed Matter and Complex Systems, 7(1999), no. 2, 293-308.

[6] R.Baeza-Yates, G.Navarro, R.Gavaldá, R.Schehing, Bounding the expected length of the longest common subsequences and forests, Theory of Computing Systems, 32(1999), no. 4, $435-452$.

[7] Kang Ning, Kwok Pui Choi, Systematic assessment of the expected length, variance and distribution of Longest Common Subsequences //arXiv preprint arXiv:1307.2796, 2013.

[8] J.D.Dixon, Longest common subsequences in binary sequences //arXiv preprint arXiv:1307.2796, 2013.

[9] S.V.Znamenskij, A picture of common subsequence length for two random strings over an alphabet of 4 symbols, Program systems: theory and applications, 7(2016), no. 1(28), 201208.

\section{Формула для средней длины длиннейшей общей подпоследовательности}

\author{
Сергей В. Знаменский \\ Институт программных систем РАН \\ Петра Первого, 4,
}

Переславльский район, Ярославская обл., 152021

Россия

Математическое ожидание Е длиннейшей общей подпоследовательности букв двух случайнъх слов рассматривается как функиия от мощности алфавита $|A|$ и длин $m$ и $n$ этих слов. При этом предполагается, что любая буква независимо и с равной вероятностъю оказывается в любой позиции слова. Предъявлено простое выражение для $E(\alpha, m, n)$ nри биксированных $\alpha u m+n$.

Ключевые слова: длиннейшая общая подпоследовательность, математическое ожидание, длина LCS, численное моделирование, асимптотическая формула 